\title{
Defining Arrow of Time at the Start of Inflation by Expansion of Entropy in a Taylor Series and Examining Initial Conditions
}

\author{
Andrew Beckwith \\ Physics Department, College of Physics, Chongqing University, Chongqing, China \\ Email: rwill9955b@gmai.com, abeckwith@uh.edu
}

How to cite this paper: Beckwith, A. (2021) Defining Arrow of Time at the Start of Inflation by Expansion of Entropy in a Taylor Series and Examining Initial Conditions. Journal of High Energy Physics, Gravitation and Cosmology, 7, 773-783.

https://doi.org/10.4236/jhepgc.2021.73045

Received: March 23, 2021

Accepted: June 1, 2021

Published: June 4, 2021

Copyright $\odot 2021$ by author(s) and Scientific Research Publishing Inc. This work is licensed under the Creative Commons Attribution International License (CC BY 4.0).

http://creativecommons.org/licenses/by/4.0/

\begin{abstract}
First, we do a Taylor series expansion of Entropy. Afterwards we define the arrow of time. After that, we define what terms we will analyze in the Taylor series expansion of entropy to help in finding initial conditions which may allow for the earliest possible identification of the Arrow of Time in cosmology. Definition of the arrow of time will allow choosing different initial starting points. That is, that in the actual equations of classical GR, there is no reason to have time asymmetry after given initial conditions. Time asymmetry is built into initial conditions and we start to explore which initial conditions may assist in evaluating contributions to Entropy via an analysis of which terms in a Taylor series survive, and what their sign and contribution values are.
\end{abstract}

\section{Keywords}

Arrow of Time, Cosmological Bounce, Information, Entropy

\section{Introduction}

This section is concerned with describing the arrow of time and initial conditions in cosmology.

In Cosmology, there is one outstanding datum, which is that in classical GR, outside of the initial conditions of the beginning of space-time, there is, in reality, no reason for the time's arrow. We will introduce the time's arrow, in the context of cosmology via initial conditions. We look at a Taylor series expansion of entropy and the relative import of terms in the series expansion in order to delineate if conditions for an arrow of time defined as early as possible in cosmology are possible. These evaluations of terms I the Taylor series expansion of entropy 
will be brought up in terms of the initial conditions of the arrow of time, which we maintain should be infidelity to the t'Hooft article's caution as to initial conditions.

\subsection{Look First at a Taylor Series Expansion of Entropy}

Doing this in terms of energy leads to:

$$
S(E)=S(\Delta E)+\left.(E-\Delta E) \frac{\mathrm{d} S(E)}{\mathrm{d} E}\right|_{E=\Delta E}+\left.\frac{(E-\Delta E)^{2}}{2} \frac{\mathrm{d}^{2} S(E)}{\mathrm{d} E^{2}}\right|_{E=\Delta E}+\text { H.O.T. }
$$

Our analysis will be using the following, i.e. we declare an arrow of time, as we define in the next section will exist if, assuming the higher-order terms are neglectable for now.

$$
\left.(E-\Delta E) \frac{\mathrm{d} S(E)}{\mathrm{d} E}\right|_{E=\Delta E}+\left.\frac{(E-\Delta E)^{2}}{2} \frac{\mathrm{d}^{2} S(E)}{\mathrm{d} E^{2}}\right|_{E=\Delta E} \geq 0
$$

We now supecify the early universe, which makes what we are doing a linkage to time, i.e.

We pick Entropy as represented by an energy term $E$, for the following reason [1] [2] [3].

Shalyt-Margolin and Tregubovich (2004, p. 73) [1], Shalyt-Margolin (2005, p. 62) [2] [3].

$$
\begin{aligned}
& \Delta t \geq \frac{\hbar}{\Delta E}+\gamma t_{P}^{2} \frac{\Delta E}{\hbar} \Rightarrow(\Delta E)^{2}-\frac{\hbar \Delta t}{\gamma t_{P}^{2}}(\Delta E)^{1}+\frac{\hbar^{2}}{\gamma t_{P}^{2}}=0 \\
& \Rightarrow \Delta E=\frac{\hbar \Delta t}{2 \gamma t_{P}^{2}} \cdot\left(1+\sqrt{1-\frac{4 \hbar^{2}}{\gamma t_{P}^{2} \cdot\left(\frac{\hbar \Delta t}{2 \gamma t_{P}^{2}}\right)^{2}}}\right)=\frac{\hbar \Delta t}{2 \gamma t_{P}^{2}} \cdot\left(1 \pm \sqrt{1-\frac{16 \hbar^{2} \gamma t_{P}^{2}}{(\hbar \Delta t)^{2}}}\right)
\end{aligned}
$$

For sufficiently small $\gamma$. The above could be represented by [3].

$$
\begin{aligned}
& \Delta E \approx \frac{\hbar \Delta t}{2 \gamma t_{P}^{2}} \cdot\left(1 \pm\left(1-\frac{8 \hbar^{2} \gamma t_{P}^{2}}{(\hbar \Delta t)^{2}}\right)\right) \\
& \Rightarrow \Delta E \approx \text { either } \frac{\hbar \Delta t}{2 \gamma t_{P}^{2}} \cdot \frac{8 \hbar^{2} \gamma t_{P}^{2}}{(\hbar \Delta t)^{2}} \text {, or } \frac{\hbar \Delta t}{2 \gamma t_{P}^{2}} \cdot\left(2-\frac{8 \hbar^{2} \gamma t_{P}^{2}}{(\hbar \Delta t)^{2}}\right)
\end{aligned}
$$

This would lead to a minimal relationship between change in $E$ and change in time as represented by Equation (4), so that we could to first order, say be looking at something very close to the traditional Heisenberg uncertainty principle results of approximately:

$$
\Delta E \approx \frac{\hbar \Delta t}{2 \gamma t_{P}^{2}} \cdot \frac{8 \hbar^{2} \gamma t_{P}^{2}}{(\hbar \Delta t)^{2}} \equiv \frac{4 \hbar}{\Delta t}
$$

Or,

$$
\Delta E \Delta t \approx 4 \hbar
$$


Assuming that we are using Equation (2) to define the genesis of an arrow of time, we by Equation (2) and Equation (6) could be defining a necessary condition for the start of an arrow of time. So first we state some particular constraints on the arrow of time, and then go to our corresponding Entropy expressions in cosmology as defined by using the results of [4], page 47 for a Rindler space representation of entropy density of saying massless bosons in "low dimensions" as:

$$
\frac{S}{L}=\frac{\pi}{3} T
$$

where $S$ is entropy, $L$ is a length, specified for a space-time lattics, and $T$ is the temperature, whereas we use the following [5] for energy, $E$ and Temperature:

$$
E=\frac{d(\text { space time }) k_{B} T}{2}
$$

If say we use Equation (6), Equation (7) and Equation (8), we could write, say the following for a hoson "gas":

$$
S=\frac{2 \pi L E}{3 d(\text { space time }) k_{B}}
$$

If so then, to first order, we have for an arrow of time, the situation where,

$$
\begin{aligned}
& \left.(E-\Delta E) \frac{\mathrm{d} S(E)}{\mathrm{d} E}\right|_{E=\Delta E}+\left.\frac{(E-\Delta E)^{2}}{2} \frac{\mathrm{d}^{2} S(E)}{\mathrm{d} E^{2}}\right|_{E=\Delta E} \\
& \approx(E-\Delta E) \frac{2 \pi L}{3 d(\text { space time }) k_{B}} \\
& \approx \frac{L(T-\Delta T)}{3} \geq 0, \text { iff } T \geq \Delta T
\end{aligned}
$$

This is for 2-dimensional space-time where we can presume $L$ approximately a Planck Length. We are having that the change in energy is related to the change in temperature $T$, due to the space time lattice having an initial temperature $T$ which would be greater than the subsequent change in temperature delta $T$ later on in the space time lattice. For forming an arrow of time, it gets worse, taking Equation (10) and isolating the time step factor, according to [4] we are looking at for an arrow of time, the situation for which we have if we employ Equation (8) for energy.

$$
\frac{\hbar}{t} \geq \frac{\hbar}{\Delta t} \Rightarrow \Delta t \geq t
$$

If $t$ is the initial time, then what this is saying is that the change in time from the initial time would have to be greater than the initial time. i.e. this seems to be specifying a one way increase in time. That may be sufficient for saying we have an arrow of entropy. But it means that we would likely have to think of $t$, in Equation (11) as a minimum time step.

If we are higher than 2 spatial dimensions, it is still very likely we will be looking at the increase in time stepping to be given by a higher dimensional analogue to Equation (11) above.

How likely would this be in terms of early universe dynamics? Before we go 
there we should review what is known about the arrow of time, and initial conditions.

Keep in mind that what we would like to consider is if there is any connection to $[5]$.

$$
\begin{gathered}
a(t)=a_{\text {initial }} t^{\gamma} \\
\rho \approx \frac{\dot{\phi}^{2}}{2}+V(\phi) \equiv \frac{\gamma}{8 \pi G} \cdot t^{2}+V_{0} \cdot\left\{\sqrt{\frac{8 \pi G V_{0}}{\gamma(3 \gamma-1)}} \cdot t\right\}^{\sqrt{\frac{\gamma}{4 \pi G}}-\sqrt{\frac{8 \pi G}{\gamma}}}
\end{gathered}
$$

As well as the Nuevo result of [6].

$$
m_{g}=\frac{\hbar}{c} \cdot \sqrt{\Lambda}
$$

Whereas we postulate a wavelength for resident DE which could lead to Equation (14).

$$
\lambda_{\mathrm{DE}} \approx 10^{30} \ell_{\mathrm{Planck}}
$$

Equation (15) giving us some version of a tie in with Dark energy as stated in [7].

And having the following counting algorithm, mainly some variant of:

$$
S(\text { initial }) \sim n(\text { graviton count })
$$

If one is looking at a thermally based definition of entropy based upon some variant of Equation (8) and also taking into account [7].

$$
\rho_{\mathrm{DE}}=\frac{\Lambda}{8 \pi G} \approx \hbar \cdot \frac{(2 \pi)^{4}}{\lambda_{\mathrm{DE}}^{4}}
$$

Where one may have an interrelationship of Equation (17) with Equation (14) and say counting of resultant gravins, is dependent upon temperature, then what happens to the arrow of time if there is a decrease in temperature, not a decrease?

We are then obviously going to have problems because traditional inflationary theory has that there is a drop off of temperature, right after the initiation of inflation that this is going to make connections to a temperature based generation of entropy, where we could have, say Equation (8) above for temperature dependence, or even Entropy the cube of Temperature very hard to link as to the Arrow of time, especially IF the start to the universe is due to ultra high temperatures which then cool.

\subsection{Generic Arrow of Time Defined with Heuristics}

First of all consider the quote given by Eddington which states some of the problem.

Let us draw an arrow arbitrarily. If as we follow the arrow we find more and more of the random element in the state of the world, then the arrow is pointing towards the future, if the random element decreases the arrow points towards the past. That is the only distinction known to physics. This follows at once if 
our fundamental contention is admitted that the introduction of randomness is the only thing which cannot be undone. I shall use the phrase "time's arrow" to express this one-way property of time which has no analogue in space [8].

In a word we have that the entire discussion of entropy, its production, and all that start with the $2^{\text {nd }}$ law of thermodynamics [8], which we can simply state as:

$$
\frac{\mathrm{d} S \text { (entropy) }}{\mathrm{d} t} \geq 0
$$

Whereas the question raised, in [8] can be rendered in the following.

This law is certainly not symmetric in time; if we interchanged past and future the entropy would tend to decrease. How did we get, from basic reversible equations to a manifestly irreversible result?

As a given, we may consider what it takes to form initial conditions. One thought to keep in mind is that we will be when establishing an order of time be affected, as brought up by t'Hooft [9].

If we adhere to the quantum mechanical description of all microscopical dynamical laws, we find the CPT theorem on our way, which implies that if we combine time reversal $\mathrm{T}$ with parity reversal $\mathrm{P}$ and particle-antiparticle interchange $\mathrm{C}$, then this symmetry is perfect. We could well stick to our verdict that Nature's boundary conditions in the time direction suffice to explain the arrow of time.

In a word, we get times ARROW of time, going back to the ideas of Eddington [5], and [5] as a consequence of how we choose the initial conditions. To do so we first of all start with the initial.

\section{Methods}

We examine in this section several different cosmological models.

At the moment of the Big Bang, almost all of the entropy was due to radiation, and the total entropy of the Universe was about $S=1088 \mathrm{kB}$, or slightly higher.

There was a sea of particles, including matter, antimatter, gluons, neutrinos and photons, all around at energies billions of times higher than what the LHC can obtain today. There were so many of them-perhaps $10^{90}$ in total, if there was a traditional model of the big bang and inflation [10].

$$
S \sim 3 \frac{m_{\text {Plank }}^{2}\left[H=1.66 \cdot \sqrt{\tilde{g}_{*}} \cdot T^{2} / m_{\text {planck }}\right]^{2}}{T} \sim 3 \cdot\left[1.66 \cdot \sqrt{\tilde{g}_{*}}\right]^{2} T^{3}
$$

If we have a beach ball sized "universe" at the end of the inflationary era, with say temperature of $T$ proportional to Planck temperature, of $T$ 1.416785(71) $\times 10^{32}$ kelvin we can approach $S=10^{88} k_{B}$ On the other hand, we may have a value slightly larger. Is this due to thermal versus particle generation? If there was a traditional model of the big bang and inflation [7] We will then have the situation which has Equation (14) holding due to superhot Planckian temperatures holding where we also would have $g_{*}$ to be the initial degrees of freedom which according to Kolb and Turner [11] would take the value of about 100 to 120 . 
To measure entropy in cosmology we can count photons. If the number of photons in a given Volume is $N$, then the entropy of that volume is $S \sim k N$ where $k$ is Boltzmann's constant.

Is there a way before the generation of the CMBR to do the same thing in terms of a counting procedure, like $S \sim k N$, with $N$ a number or count of "particles" in order to compliment Equation (14) above? Any such attempt would have to adhere to the following outline for an arrow of time.

In order to have the value of the increasing onset of the entropy we would like to have the following, namely by using Equation (18) we would assert a causal ordering following the given values of:

$$
S+\Delta S \approx n+\Delta n \geq n \text { iff } t+\Delta t \geq t
$$

The problem is, with decreasing temperature, from the initial start of inflation, this program for Equation (20) looks dubious and has to be reconciled with Equation (19) if there is a decrease in initial temperatures.

We state that Equation (19) is a multidimensional generalization of Equation (9) but it actually makes the resolution of comparing Equation (19) and Equation (20) harder, not easier.

Note that Y. Jack Ng. has [12], from a very different standpoint derived $S \sim n$ based upon string theory derived ideas, with $\mathrm{n}$ a "particle" count, which in $\mathrm{Y}$. Jack Ng's procedure is based upon the number of dark matter candidates in a given region of phase space. Y. Jack Ng's idea was partly based upon the idea of quantum "infinite" statistics, and a partition function [12].

What about Breaking up of Initial Black Holes, Right after the Birth of a New Universe?

In [10], there is a reference to the destruction of primordial black holes which is given as when the density of universe climbs to a value given as $\omega_{Q}=p_{Q} / \rho_{Q}$ is defined, with the numerator being the pressure, and denominator density of phantom fields, which leads to by [13] a density for which there is the breakup of primordial black holes.

$$
\rho_{B H} \approx M_{p}^{4} \cdot\left(\frac{M_{p}^{2}}{M^{2}}\right) \cdot\left(\frac{3}{32 \pi}\right) \cdot \frac{1}{\left|1+\omega_{Q}\right|}
$$

If the black holes are broken up lead to particle generation, which could then feed into writing say:

$$
S_{\text {bounce }} \approx n_{Q}=\text { Gravtions from black holes }
$$

The problem would then be to delineate conditions for which the Equation (21) would lead from a low to a high entropy build up, which would require a lot of computer simulation work to ascertain, but it may, if done carefully yield conditions as to the causal conditions for creation of an arrow of time;. The problem would be then to ascertain if and when the causal conditions lead to the density of the Universe yielding a value say of the order of magnitude of Equation (21) above. 
Keep in mind that according to [14] Khlopov, has the following for black hole density, namely:

$$
\rho_{B H} \approx \frac{M}{\left(r_{g}=2 G M / c^{2}\right)^{3}} \equiv \frac{c^{6}}{8 G^{3} M^{2}}
$$

Here, $M$ is the presumed mass of a black hole, and the result is counter intuitive to say the least, as $r_{g}$ is the mass of the configuration with mass $M$.

We state that in this situation we have that there may be:

$$
S_{\text {gravtions }} \approx n_{\text {gravtions }} \propto S_{\text {Thermal }} \approx T_{\text {thermal temp }}^{3}
$$

But this depends upon having:

$$
\rho_{B H} \approx \frac{c^{6}}{8 G^{3} M^{2}} \approx \frac{3 M_{P}^{6}}{32 \pi M^{2}\left|1+\omega_{Q}\right|}
$$

If we use $\left|1+\omega_{Q}\right| \approx \frac{3}{4 \pi}$ and $M_{P}=G=c=1$, we have a $\omega_{Q} \approx-\left(\frac{4 \pi-3}{4 \pi}\right)$ so that then pressure and density are approximate negative values of each other, which is implying the following. i.e., The cosmological constant has negative pressure, but positive energy. The negative pressure ensures that as the volume expands then matter loses energy (photons get red shifted, particles slow down); this loss of energy by matter causes the expansion to slow down, but the increase in energy of the increased volume is more important. The increase of energy associated with the extra space the cosmological constant fills has to be balanced by a decrease in the gravitational energy of the expansion-and this expansion energy is negative, allowing the universe to carry on expanding.

\section{Comparing Times Arrow}

We consider release of information and compare it with Seth Lloyd's linkage of entropy and bits of information, as in reference [15] obtained the following and this is to a certain degree duplicated in our work but it has limitations.

A way to obtain traces of information exchange, from prior to present universe cycles is finding linkage between information and entropy. If such a parameterization can be found and analyzed, then Seth Lloyd's [15] shorthand for entropy,

$$
I=S_{\text {total }} / k_{B} \ln 2=[\# \text { operations }]^{3 / 4}=\left[\rho \cdot c^{5} \cdot t^{4} / \hbar\right]^{3 / 4}
$$

could be utilized as a way to represent information which can be transferred from a prior to the present universe. The question to ask, if does Equation (26) permit a linkage of gravitons as information carriers, and can there be a linkage of information, in terms of the appearance of gravitons in the time interval of, say $0<t<t_{\text {Planck }}$ either by vacuum nucleation of gravitons/information packets Oops. What is the problem? No special initial conditions as specified by "tHooft" in [9] in the setup of an initial arrow of time configuration. Equation (26) is completely general, and does not tie in with also how we can have a satisfaction as to Equation (11) and Equation (20) given above. 


\section{Conclusions}

It is a much harder problem than what most physics people think that of satisfying all of the arrows of times constituent parts. In the 1980s, Hawking [16] in his 1985 in his paper specifically also added a continually expanding volume of space-time as a reset of initial conditions for an arrow of time. However, in the Hawking problem, we do not have the special initial conditions for the arrow of time, and in addition, if there is a singularity we have the problem of peak to decreasing $\mathrm{T}$ values, temperature, which vexes present cosmological models. In which then new thinking will be required, that will be difficult for a lot of cosmologists to accept. And even good cosmologists as in [17], Linde come up with what I regard as fanciful suggestions in a field which has still not enough data and work behind it, to falsify our ideas with concrete data In [17] its author comes up with a suggested likelihood of the Cosmological constant having its present value based upon the Hartle-Hawking wavefunction of the universe, involving taking the actual exponential of a negative of the Hartle Hawking wavefunction of the universe. In doing so he obtained having a given value of $\Lambda$ via Hartle-Hawking theory having a given probability of the square of the HartleHawking wavefunction, i.e.,

$$
P(\text { probability }) \sim \exp \left(-24 \pi^{2} / \Lambda\right)=\exp \left(-S_{A}\right)
$$

This probability would lead to a ridiculously large time value one would have to wait for any such occurrence happening with a time of a value infinitely larger than the age of the expected universe.

$$
t \sim \exp \left(S_{A}\right) \sim 10^{10^{12}}
$$

In short, we can and must do better than this. And this requires new models and geometric paradigms to access what we may eventually be able to vet via experimental data sets.

For the record, I have read in detail [18] and used a part of his ideas in the discussion of deformed special relativity and quantum uncertainty. I also was cognizant of [19] and nearly used it, but stopped when the author was intent upon using a version of entropy that automatically mandates nonexistent entropy at the very start of the expanding universe. In so many words, the jury is out on that one and there may be a different venue that shows up later.

\section{Discussion}

What of the No Boundary Condition and the Arrow of Time Hypothesis by Hawking?

In [20] Hawking gave this condition, on page 335, that of Equation (29) as a constant value.

$$
A a^{\tilde{p}}=\text { Cons }
$$

where $A$ is the amplitude of a "perturbation", and we pick a to be the scale factor of the universe, with the value of $a_{\text {Today }}=1$, and at the start of inflation we could 
have initial $a_{\text {initial }} \leq 10^{-25}$. I.e. if we do so, we then have, via this mechanism a way to make a statement as to initial conditions for defining an arrow of time.

The problem in 2 dimensions, is that we have as given in Equation (11) of needing $\Delta t \geq t$ to define condtions for an Arrow of time.

i.e. giving say a time increment of $\Delta t \approx t_{\text {Planck }}=$ Planck time, we are specifying looking at:

$$
\begin{aligned}
a(t) & \approx a(\Delta t)+(t-\Delta t) \cdot\left[\frac{\mathrm{d} a}{\mathrm{~d} t}\right]_{t=\Delta t}+H . O . T \\
& \approx a_{\text {initial }} \cdot\left[(\Delta t)^{\tilde{\gamma}}+\tilde{\gamma}(t-\Delta t) \cdot t^{\tilde{\gamma}-1}\right]
\end{aligned}
$$

When applying this to Equation (29) we are looking at:

$$
\begin{aligned}
& A a^{\tilde{p}} \approx A \cdot\left(a_{\text {initial }} \cdot\left[(\Delta t)^{\tilde{\gamma}}+\tilde{\gamma}(t-\Delta t) \cdot t^{\tilde{\gamma}-1}\right]\right)^{\tilde{p}}=\text { Cons } \\
& a_{\text {Today }}=1
\end{aligned}
$$

Whereas we can have situations for which if we are looking at Equation (11) for having an arrow of time with say a 2 dimensional initial grid of space-time when $\Delta t \geq t$, we could have a net shrinkage, not increase in spatial expansion, of initial geometry, even if there is $\Delta t \geq t$, for say $\Delta t$ of the order of Planck time, and $t$ smaller than Planck Time.

The point is this, in the case of Equation (31) to be consistent with the arrow of time, as given by [16]. where Hawking specified an arrow of time exists with an increase of space-time volume as we go from time $t$ to $t+\Delta t$ as an additional condition for showing the existence of an arrow of time, we are still specifying highly restrictive conditions upon the coefficient of $\tilde{p}$, i.e. depending upon the geometry of initial space-time evaluated, even if $\Delta t \geq t$ is not required due to different initial geometry.

The point being this, in cosmology, there is no easy way to always initially satisfy Equation (20) if we deviate say from an incremental step in time being evaluated by Equation (31).

The mess gets even worse if we look at the purported "wavefunction of the Universe" as specified by [21], page 273 for a Wheeler De Witt wavefunction of the universe, which is,

$$
\begin{aligned}
& \frac{\mathrm{d}^{2} \psi}{\mathrm{d} \tilde{z}^{2}}+\hat{a}^{2}\left(\tilde{z}^{4}-\tilde{z}^{2}\right) \psi=0 \\
& \tilde{z}=a(t) / a_{\text {initial }}, \hat{a}=\frac{9 \pi c^{5}}{2 \hbar G \Lambda}
\end{aligned}
$$

$$
\Rightarrow \psi=\frac{\tilde{N}_{0} \cos \left(\left|\frac{\hat{a}}{3}\left(\tilde{z}^{2}-1\right)^{3 / 2}\right|-\frac{\pi}{4}\right)}{\left|\hat{a}\left(\tilde{z}^{4}-\tilde{z}^{2}\right)^{1 / 2}\right|^{1 / 2}}
$$

This wavefunction would vanish if there is NO length initially from a spacetime singularity, but the point of fact is that Equation (31) is giving very imprecise information as to initial conditions, to begin with. And we do have that 
problem of satisfying "tHoofs" conditions for the arrow of time, as in [9] which specified NOT using general solutions, only particular solutions to the arrow of time being specified as early as possible in space-time.

All of which contravenes the spirit of Equation (11) and Equation (24) and refinements of the cosmological conditions satisfying the initial conditions may await further development along the lines of Chapter 5, Schrodinger Maps, pp 201-222 in [21].

We have when writing this avoided going beyond traditional Cosmological theory and what we have tried to indicate is what the shortcomings of the traditional cosmological models due to the imprecision of their initial conditions which may allow us to satisfy [9] in the future developments.

\section{Conclusion with Wrap up Discussions}

1) Traditional cosmology has major problems in identifying initial conditions as to [9] criteria as to what constitutes an arrow of time, with expanding spacetime, iternation of time steps, and counting algorithms all reconciled with traditional nomenclature as given in [11] as to temperature-dependent growth of entropy, initially, because of the datum seen in cosmology that the initial spacetime temperature, T, DECREASES during inflation. See [11]. Worse than that, traditional counting algorithms of cosmology, as far as the creation of countable "wave-particles" as presently constituted in orthodox comologies are post inflationary phenomenon. i.e. using the traditional models would imply that we can only form the arrow of time, POST inflation.

2) The problem of having in fildelity entropy growth with causal relationships specified in the beginning an additive nature to Entropy as it is consistent with forming the initial arrow of time is a vexing one and which means reconciling Equation (I1) and Equation (24) with particular initial condtions.

3) We state for the record that Seth Lloyds attempt, as given in Equation (26) contravenes [9].

4) New physics and new models have to be found. The author rejects what seems to be given in the traditional cosmological models implying that the arrow of time cannot be defined at the start of inflation itself.

\section{Conflicts of Interest}

The author declares no conflicts of interest regarding the publication of this paper.

\section{References}

[1] Shalyt-Margolin, A.E. (2006) Deformed Density Matrix and 21 Quantum Entropy of the Black Hole. Entropy, 8, 31-43. https://doi.org/10.3390/e8010031

[2] Shalyt-Margolin, A.E. (2005) The Density Matrix Deformation in Physics of the Early Universe and Some of Its Implications. Quantum Cosmology Research Trends. Horizons in World Physics, 246, 49-91.

[3] Shalyt-Margolin, A.E. and Tregubovich, A.Y. (2004) Deformed Density Matrix and Generalized Uncertainty Relation in Thermodynamics. Modern Physics Letters A, 
19, 71-81. https://doi.org/10.1142/S0217732304012812

[4] Susskind, L. and Lindsay, J. (2005) An Introduction to Black Holes, Information and the String Theory Revolution. World Scientific Press, Singapore.

https://doi.org/10.1142/5689

[5] Thanu, P. (2006) An Invitation to Astrophysics. World Scientific Press, World Scientific Series in Astronomy and Astrophysics: Volume 8. Singapore.

https://doi.org/10.1142/6010

[6] Li, M., Li, X.-D., Wang, S. and Wang, Y. (2015) Dark Energy. Peking University Press, World Scientific, Singapore.

[7] Novello, M. and Neves, R.P. (2003) The Mass of the Graviton and the Cosmological Constant. Classical and Quantum Gravity, 20, L67-L73. https://doi.org/10.1088/0264-9381/20/6/101

[8] Friedel, W. (2005) The Scientist as Philosopher: Philosophical Consequences of Great Scientific Discoveries. Springer, 143 p.

[9] 't Hooft, G. (2018) Time, the Arrow of Time and Quantum Mechanics. Frontiers in Physics, 6, 81. https://doi.org/10.3389/fphy.2018.00081 https://www.frontiersin.org/articles/10.3389/fphy.2018.00081/full

[10] Beckwith, A.W. (2018) Initial Conditions for Defining an Arrow of Time at the Start of Inflation? Journal of High Energy Physics, Gravitation and Cosmology, 4, 787-795. https://doi.org/10.4236/jhepgc.2018.44044

[11] Kolb, E. and Turner, S. (1994) The Early Universe. Westview Press, Chicago, USA,

[12] Ng, Y.J. (2008) Spacetime Foam: From Entropy and Holography to Infinite Statistics and Nonlocality. Entropy, 10, 441-461. https://doi.org/10.3390/e10040441

[13] Freeze, K., Brown, M. and Kinney, W. (2012) The Phantom Bounce, A new proposal for an oscillating Cosmology. The Arrows of Time, 172, 149-156.

https://doi.org/10.1007/978-3-642-23259-6_7

[14] Kohopov, M. (2012) Fundamentals of Cosmic Particle physics. Cambridge International Science Publishing, Cambridge, UK.

[15] Seth, L. (2002) Computational capacity of the universe. Physical Review Letters, 88, Article ID: 237901. https://arxiv.org/pdf/quant-ph/0110141.pdf https://doi.org/10.1103/PhysRevLett.88.237901

[16] Hawking, S.W. (1985) Arrow of Time in Cosmology. Physical Review D, 32, Article No. 2489. https://doi.org/10.1103/PhysRevD.32.2489

[17] Linde, A. (2007) The inflationary Multiverse. In: Carr, B., Ed., Universe or Multiverse, Cambridge University Press, New York, 127-149.

https://doi.org/10.1017/CBO9781107050990.010

[18] Shalyt-Margolin, A. (2010) Entropy in the Present and Early Universe: New Small Parameters and Dark Energy Problem. Entropy, 12, 932-952.

[19] Luongo, O. and Quevedo, H. (2012) Cosmographic Study of the Universe's Specific Heat: A Landscape for Cosmology? https://arxiv.org/abs/1211.0626

[20] Stephen, H. (1994) The No Boundary Condition and the Arrow of Time. In: Hawiwell, J.J., Perez-Mercarder, J. and Zurek, W.H., Eds., Physical Origins of Time Asymmetry, Cambridge University Press, Cambridge, UK, 346-356

[21] Dalarsson, M., and Dalarsson, N. (2005) Tensors, Relativity and Cosmology. Elsevier Academic Press, London, United Kingdom.

[22] Koch, H., Tataru, D. and Visan, M. (2014) Dispersive Equations and Nonlinear Waves. Birkhauser, New York. https://doi.org/10.1007/978-3-0348-0736-4 